\title{
Fourier Pseudospectral Solution for a 2D Nonlinear Paraxial Envelope Equation of Laser Interactions in Plasmas
}

\author{
Abdelrahman I. Mahdy \\ Plasma and Nuclear Fusion Department, Nuclear Research Centre, Atomic Energy Authority, Cairo, Egypt \\ Email: aimahdy@yahoo.com
}

How to cite this paper: Mahdy, A.I. (2016) Fourier Pseudospectral Solution for a 2D Nonlinear Paraxial Envelope Equation of Laser Interactions in Plasmas. Journal or Applied Mathematics and Physics, 4, 21862202.

http://dx.doi.org/10.4236/jamp.2016.412213

Received: October 19, 2016

Accepted: December 17, 2016

Published: December 20, 2016

Copyright $\odot 2016$ by author and Scientific Research Publishing Inc. This work is licensed under the Creative Commons Attribution International License (CC BY 4.0). http://creativecommons.org/licenses/by/4.0/

\begin{abstract}
We apply a Fourier pseudospectral algorithm to solve a $2 \mathrm{D}$ nonlinear paraxial envelope-equation of laser interactions in plasmas. In this algorithm, we first use the second order Strang time-splitting method to split the envelope-equation into a number of equations, next we spatially discrete the filed quantity and its spatial derivatives in these equations in term of Fourier interpolation polynomials (FFT), finally we sequentially integrate the resultant equations by means of a discrete integration method in order to obtain the solution of the envelope-equation. We carry out several numerical tests to illustrate the efficiency and to determine accuracy of the algorithm. In addition, we conduct a number of numerical experiments to examine its performance. The numerical results have shown that the algorithm is highly efficient and sufficiently accurate to solve the $2 \mathrm{D}$ envelope-equation, furthermore, it yields an optimal performance in simulating fundamental phenomena in laser interactions in plasmas.
\end{abstract}

\section{Keywords}

Laser Plasmas Interactions, Laser Envelope-Equation, Fourier Pseudospectral Method, Nonlinear Schrödinger Equation

\section{Introduction}

The envelope-equation is the equation that describes the evolution of the slowly varying laser-envelope under the static transformation of the laser coordinate (Quasi Statics Approximation QSA) [1]. In laser interactions in plasmas, the envelope-equation is widely employed to study a number of fundamental phenomena, such as self-focusing [2], parametric instability [3], modulation instability [4], laser-based acceleration [5], 
high-order harmonic generation [6], and filamentation of femtosecond laser in air plasma [7]. Over decades of noteworthy studies, the envelope-equation has succeeded to present an elaborate analysis for these phenomena at varying interactions conditions, furthermore, the equation has shown a great potential for illustrating advanced phenomena in this interactions.

Since the early studies of laser interactions in plasmas, the envelope-equation has been subject to several analytical attempts. Preliminary, the Source-Dependent Expansion (SDE) was proposed to analyze the equation, also the ray tracing was applied to follow the frequency evolution and wave number in the first order envelope-equation, as well the variation approach was used to construct an exact Hamiltonian formulation for the high order envelope-equation. It has been noted that these attempts present an insufficient analysis for the multi dimensional phenomena of the high frequency and high intensity filed, besides it comes with an ambiguous interpretation for strongly nonlinear phenomena as plasmas turbulence and plasma complexity. Because of this incomplete analysis and in order to improve the interpretation, the numerical modeling has been considered.

Over the last few years, various numerical models have been applied to solve the envelope-equation, such as the finite difference time domain (FD) [8], the standard and advanced Peceaman Rachford ADI [9], the direct integral [10], the Quasi-PIC [11], the envelope-kinetic scheme [12], the fluid three wave model [13], and the spectral method [14]. In the spectral method, the solution is approximated by a series of expansions using a trial function with a number of degrees in space and time. For examples the tau function is used as a trail function is the Tau spectral method, the Chebyshev polynomials in the Chebyshev spectral method, the Hermite function in the Hermite pseudospectral method, and the Fourier series in the Fourier pseudospectral method. It has been realized that, among the mentioned spectral approximations, the Fourier pseduospectral is most suited method to solve the envelope-equation.

The Fourier pseudospectral method has been verified as an accurate and effective technique for solving the envelope-equation in nonlinear optics [15], soliton physics [16], Bose-Einstein condensates [17], and plasma physics [18], therefore in the present article we apply a Fourier pseudospectral algorithm to the solve a 2D paraxial envelopeequation of laser interactions in plasmas. The article in organized as follow: In Section 2, we present the envelope-equation with its main mathematical approximations and physical assumptions. In Section 3, we describe the Fourier pseudospectral algorithm. In particular, we explain the solution procedures and introduce the boundary conditions. In Section 4, we illustrate the efficiency and determine the accuracy of the algorithm through several benchmark convection tests. Finally in Section 5, we examine the performance of the algorithm in a series of numerical experiments.

\section{The Envelope Equation}

The following is the envelope-equation that is employed to study the laser interactions in plasmas: 


$$
2 i \frac{\partial a}{\partial t}+\left(D_{x x}+D_{y y}\right) a+N(a) a=0
$$

As seen above, the equation is a $2 \mathrm{D}$ Nonlinear Schrödinger Equation-type (2D NLSE-type), where $a$ is vector potential, $t$ is the time, $N(a)=(1-n / \gamma)$ is the nonlinear source term, $n$ is the electron density, and $\gamma=\sqrt{1+|a|}$ is the relativistic factor. The envelope-equation is derived using three distinct approximations: first, the Quasi Static Approximation (QSA) [19] that assumes the laser evolution in time and space is much larger than the typical time and space of the plasma respond; second, the slowly varying approximation in which the envelope amplitude changes much less than $\left(\partial a / \partial t \ll\left|\omega_{0} a\right|\right)$ the laser carrier frequency $\omega_{0}$; third, the paraxial approximation that considers no variation along the direction of propagation $z(\partial / \partial z=0)$. Regarding the physical assumptions, the above equation presents a fluid description for laser interactions in underdense and collisions less plasma, in addition, in this equation two nonlinearities; which are the ponderomotive nonlinearity that is represented by $n$ and the relativistic nonlinearity that it is given by $\gamma$, are considered, while the wake nonlinearity is ignored.

\section{The Fourier Pseudospectral Algorithm}

As a matter of fact, the Fourier pseudospectral algorithm (FPSA) is a modified approach for the standard Fourier Pseudospectral method [20]. To explain this context, in the standard spectral method the solution procedures for any envelope-equation are almost started by expanding the field quantity $a$ and its spatial derivatives in this equation in term of Fourier interpolation polynomials, but in our FPSA, we initially split the envelope-equation into a number of equations using the Strang time-splitting method before carrying out the expansion. The particular purpose of this splitting is to transfer the envelope-equation into simple equations where the physical variables; the nonlinear source term $(N(a))$ and the spatial operators $\left(D_{x x}, D_{y y}\right)$ are separated, and hence more simple solution procedures can be carried out. The Strang time-splitting process for the envelope-equation and the solution procedures including the boundary conditions of the resultant equations are explained in this section.

\subsection{The Strang Time-Splitting}

Within the Strang time-splitting method, the envelope-equation can be optionally splitted into two/three equations, the two equations splitting is called the first order Strang time-splitting, while the three equations splitting is called the second order Strang time-splitting. It has been found that the second order splitting provides convergence accuracy when it is applied to the Shrödinger equation (the envelope-equation) [21], thus in our algorithm we consider the second order Strang time-splitting to split the envelope-equation.

Using the second order Strang time-splitting, our envelope-equation is splitted into the following equations: 


$$
\begin{aligned}
& 2 i \frac{\partial a}{\partial t}+N(a) a=0, \\
& 2 i \frac{\partial a}{\partial t}+D_{x x} a+D_{y y} a=0, \\
& 2 i \frac{\partial a}{\partial t}+N(a) a=0 .
\end{aligned}
$$

As noted, the above equations are classified into two sets of equations: a) two ordinary differential equations (ODE); Equations (2) and (4); which include the source term, b) one 2D partial differential equation (PDE); Equation (3), that contains the spatial operators only. Each set defines different physics, and hence each requires different numerical solution procedures as it will be explained in the coming part.

\subsection{The Solution Procedures}

In the first place, we carry out the solution procedures of Equations (2)-(4) in the time domain $t=n \Delta t$; where $\Delta t$ is the time-step size and $n$ is the number of time steps, and in the $2 \mathrm{D}$ spatial domain $\left[-L_{x}, L_{x}\right] \times\left[-L_{y}, L_{y}\right]$; where $L_{x}=m_{1} \Delta x, L_{y}=m_{2} \Delta y$, $\Delta x$, and $\Delta y$ are the grid size and $m_{1}, m_{2}$ are the maximum number of grids along $\pm x-$ and $\pm y$ - direction, receptively. In our procedures, we consider $L_{x}=L_{y}=L$, $\Delta x=\Delta y=h$, and $m_{1}=m_{2}=m$, upon that, at any time step $n$ Equations (2)-(4) can be re-written in the spatial domain as[21]

$$
\begin{aligned}
& 2 i \frac{a_{i, j}^{*}-a_{i, j}^{n}}{\Delta t}+N\left(a_{i, j}^{n}\right) a_{i, j}^{*}=0, \\
& 2 i \frac{a_{i, j}^{* *}-a_{i, j}^{*}}{\Delta t}+D_{x x} a_{i, j}^{* *}+D_{y y} a_{i, j}^{* *}=0, \\
& 2 i \frac{a_{i, j}^{n+1}-a_{i, j}^{* *}}{\Delta t}+N\left(a_{i, j}^{* *}\right) a_{i, j}^{n+1}=0,
\end{aligned}
$$

for

$$
i=-m_{1},-m_{1}+1, \cdots,-2,-1,0,1,2, \cdots, m_{1}-1, m_{1},
$$

and

$$
j=-m_{2},-m_{2}+1, \cdots,-2,-1,0,1,2, \cdots, m_{2}-1, m_{2} .
$$

Equations (5)-(7) are the final splitted form of the envelope-equation, and its solution procedures are entirely explained below.

The solution procedures of Equations (5)-(7) start at the time step $n$ in order to obtain $a^{n+1}$ in the time interval $\left[t_{n}=n \Delta t, t_{n+1}=(n+1) \Delta t\right]$ through the following three sequential steps:

Equation (5) is solved to obtain $a_{i, j}^{*}$ based on the initial input $a_{i, j}^{n}$.

As it is ordinary differential equation, Equation (5) has the following straight forward solution:

$$
a_{i, j}^{*}=\sum_{i=-m}^{m} \sum_{j=-m}^{m} \mathrm{e}^{i N\left(a_{i, j}^{n}\right) \frac{\Delta t}{2}} a_{i, j}^{n} .
$$


Equation (6) is solved to obtain $a_{i, j}^{* *}$ based on the initial input $a_{i, j}^{*}$.

To obtain the solution of Equation (6), in the beginning the field quantities $a_{i, j}^{* *}$ and $a_{i, j}^{*}$ in this equation are expanded and transfered to the wave number domain ( $\hat{a}_{i, j}^{* *}$ and $\hat{a}_{i, j}^{*}$ ) using the Discrete Fast Fourier Transform (DFT) as

$$
\begin{aligned}
& \hat{a}_{\mu, \beta}^{*}=\sum_{i=-m}^{m} \sum_{j=-m}^{m} a_{i, j}^{*} \mathrm{e}^{-i\left(k_{\mu} x_{i}\right)} \mathrm{e}^{-i\left(k_{\beta} y_{j}\right)} \\
& \hat{a}_{\mu, \beta}^{* *}=\sum_{i=-m}^{m} \sum_{j=-m}^{m} a_{i, j}^{* *} \mathrm{e}^{-i\left(k_{\mu} x_{i}\right)} \mathrm{e}^{-i\left(k_{\beta} y_{j}\right)},
\end{aligned}
$$

for

$$
\mu=-m,-m+1, \cdots,-2,-1,0,1,2, \cdots, m-1, m,
$$

and

$$
\beta=-m,-m+1, \cdots,-2,-1,0,1,2, \cdots, m-1, m \text {. }
$$

where $k_{\mu}=\pi \mu / L$ and $k_{\beta}=\pi \beta / L$ are the wave numbers along $\pm x-$ and $\pm y-$ direction, receptively. As a consequence to the above transformation, the spatial operators become

$$
\begin{aligned}
& D_{x x} \hat{a}_{\mu, \beta}^{* *}=-k_{\mu}^{2} \sum_{i=-m}^{m} \sum_{j=-m}^{m} a_{i, j}^{* *} \mathrm{e}^{-i\left(k_{\mu} x_{i}\right)} \mathrm{e}^{-i\left(k_{\beta} y_{j}\right)}, \\
& D_{x x} \hat{a}_{\mu, \beta}^{* *}=-k_{\mu}^{2} \hat{a}_{\mu, \beta}^{* *},
\end{aligned}
$$

and

$$
\begin{aligned}
& D_{y y} \hat{a}_{\mu, \beta}^{* *}=-k_{\beta}^{2} \sum_{i=-m}^{i=m} \sum_{j=-m}^{j=m} a_{i, j}^{* *} \mathrm{e}^{-i\left(k_{\mu} x_{i}\right)} \mathrm{e}^{-i\left(k_{\beta} y_{j}\right)}, \\
& D_{y y} \hat{a}_{\mu, \beta}^{* *}=-k_{\beta} \hat{a}_{\mu, \beta}^{* *} .
\end{aligned}
$$

Then, Equations (8) and (9) are substituted in Equation (6) to give

$$
2 i \frac{\hat{a}_{\mu, \beta}^{* *}-\hat{a}_{\mu, \beta}^{*}}{\Delta t}-\left(k_{\mu}^{2}+k_{\beta}^{2}\right) \hat{a}_{\mu, \beta}^{* *}=0 .
$$

Equation (10) has the following solution:

$$
\hat{a}_{\mu, \beta}^{* *}=\sum_{\mu=-m \beta=-m}^{m} \sum^{m} \mathrm{e}^{-i\left(k_{\mu}^{2}+k_{\beta}^{2}\right) \frac{\Delta t}{2}} \hat{a}_{\mu, \beta}^{*} .
$$

In the above equation, the solution is presented in the wave number domain. Therefore, we have to transfer it back to the physical domain using the Inverse DFT as

$$
\begin{aligned}
a_{i, j}^{* *} & =\frac{1}{(2 m)^{2}} \sum_{\mu=-m \beta=-m}^{m} \sum^{m} \mathrm{e}^{-i\left(k_{\mu}^{2}+k_{\beta}^{2}\right) \frac{\Delta t}{2}} \hat{a}_{\mu, \beta}^{*} \mathrm{e}^{i\left(k_{\mu} x_{i}\right)} \mathrm{e}^{i\left(k_{\beta} y_{j}\right)}, \\
i & =-m,-m+1, \cdots,-2,-1,0,1,2, \cdots, m-1, m, \\
j & =-m,-m+1, \cdots,-2,-1,0,1,2, \cdots, m-1, m .
\end{aligned}
$$

Equation (7) is solved to obtain $a_{i, j}^{n+1}$ based on the initial input $a_{i, j}^{* *}$.

Similarly to Equation(5), Equation (7) is ODE, so it has the solution given below 


$$
a_{i, j}^{n+1}=\sum_{i=-m}^{i=m} \sum_{j=-m}^{j=m} \mathrm{e}^{i N\left(a_{i, j}^{* *}\right) \frac{\Delta t}{2}} a_{i, j}^{* *}
$$

The time step $n$ is constantly increment by one and the above three steps are sequentially repeated unit we reach the maximum computational time and allocate the field quantity $a_{i, j}$ in each time step.

\subsection{The Boundary Conditions}

In the FPSA; as in the other spectral methods, the boundary conditions are restricted to be periodic, in addition, by these conditions the physical property of the laser-envelope should be preserved for a very long evolution time. To comply with these requirements, we introduce the following boundary conditions:

$$
\begin{aligned}
& a(-L, y, t)=a(L, y, t)=0 \quad \text { for } \quad-L \leq y \leq L \text {, and } \quad t \geq 0 \text {, } \\
& a(x,-L, t)=a(x, L, t)=0 \text { for }-L \leq x \leq L \text {, and } t \geq 0 \text {, }
\end{aligned}
$$

and

$$
\begin{array}{ll}
\frac{\partial}{\partial x} a(-L, y, t)=\frac{\partial}{\partial x} a(L, y, t)=0 & \text { for } \quad-L \leq y \leq L, \text { and } \quad t \geq 0 \\
\frac{\partial}{\partial y} a(x,-L, t)=\frac{\partial}{\partial y} a(x, L, t)=0 & \text { for } \quad-L \leq x \leq L, \text { and } \quad t \geq 0 .
\end{array}
$$

As it is clear above, the boundary conditions are periodic. Furthermore, within these conditions, the soliton solution is valid. It is necessary to know that, in the soliton solution the field quantity $a$ and its spatial derivatives are vanished at $\pm L$, at this circumstance, the laser energy is always consuming on the envelope and absorbing in the boundaries, which is the property has to be kept preserved over the time.

\section{Numerical Tests}

The numerical test is fundamentally required in order to illustrate the efficiency and to determine the accuracy of the FPSA, therefore in this section we conduct this test. In fact, we perform two tests, in the first test we benchmark the algorithm against the analytical solution of a 1D Cubic Nonlinear Shrödinger Equation [22] to illustrate the efficiency, and in the second one we apply the algorithm to evaluate the absolute error of a 2D Nonlinear Shrödinger Equation to determine the accuracy. The details of the two tests are presented in the following subsections.

\subsection{Illustrating the Efficiency}

The equation given below is the Cubic Nonlinear Shrödinger Equation (CNLS).

$$
i \frac{\partial u}{\partial t}+\frac{\partial^{2} u}{\partial x^{2}}+q|u|^{2} u=0 \quad x \Subset R \quad t \geq 0 .
$$

The CNLS is a general envelope-equation that describes critical phenomena in plasma, such as the optical propagation in dispersive medium, the waves generation, and the self-focusing of laser beam. As shown above, the equation is $1 \mathrm{D}$ time-dependent, 
where $u$ is the complex amplitude, $\left(\partial^{2} u / \partial x^{2}\right)$ is the spatial derivative which governs the nonlinear effect, and $\left(q|u|^{2} u\right)$ is the cubic term that maintains the dispersion phenomena. As known, the CNLS presents the solution when the nonlinear effect is balanced with the dispersion phenomena, at this balance both of the single soliton solution, the multi-soliton solution, and the boundary soliton solution are valid. Among these valid solutions, we selected the single soliton solution of the following analytical solution to run the current test:

$$
u(x, t)=\sqrt{2 \alpha / q} \exp \left\{i\left[\frac{1}{2} c x-\left(\frac{1}{4} c^{2}-\alpha\right) t\right]\right\} \sec h\{\sqrt{\alpha}(x-c t)\} .
$$

To run the test, we numerically solved the CNLS using the FPSA in the space domain $-40 \leq x \leq 40$, for $\alpha=1, q=1$ and $c=1$, with the following initial profile:

$$
u(x, 0)=\sqrt{2 \alpha / q} \exp \left(\frac{1}{2} i c x\right) \sec h\{\sqrt{\alpha x}\},
$$

and the following boundary conditions:

$$
u(x, t)=0 \quad \text { at } \quad x=-L, L, \quad \text { and } \quad t \geq 0 .
$$

To illustrate the efficiency, we plotted the analytical solution together with the obtained numerical solution at different grid sizes in Figure 1 and at different time-step sizes in Figure 2. In theses figures, the analytical solution is plotted in solid-lines and the numerical solution is given in dashed-lines.
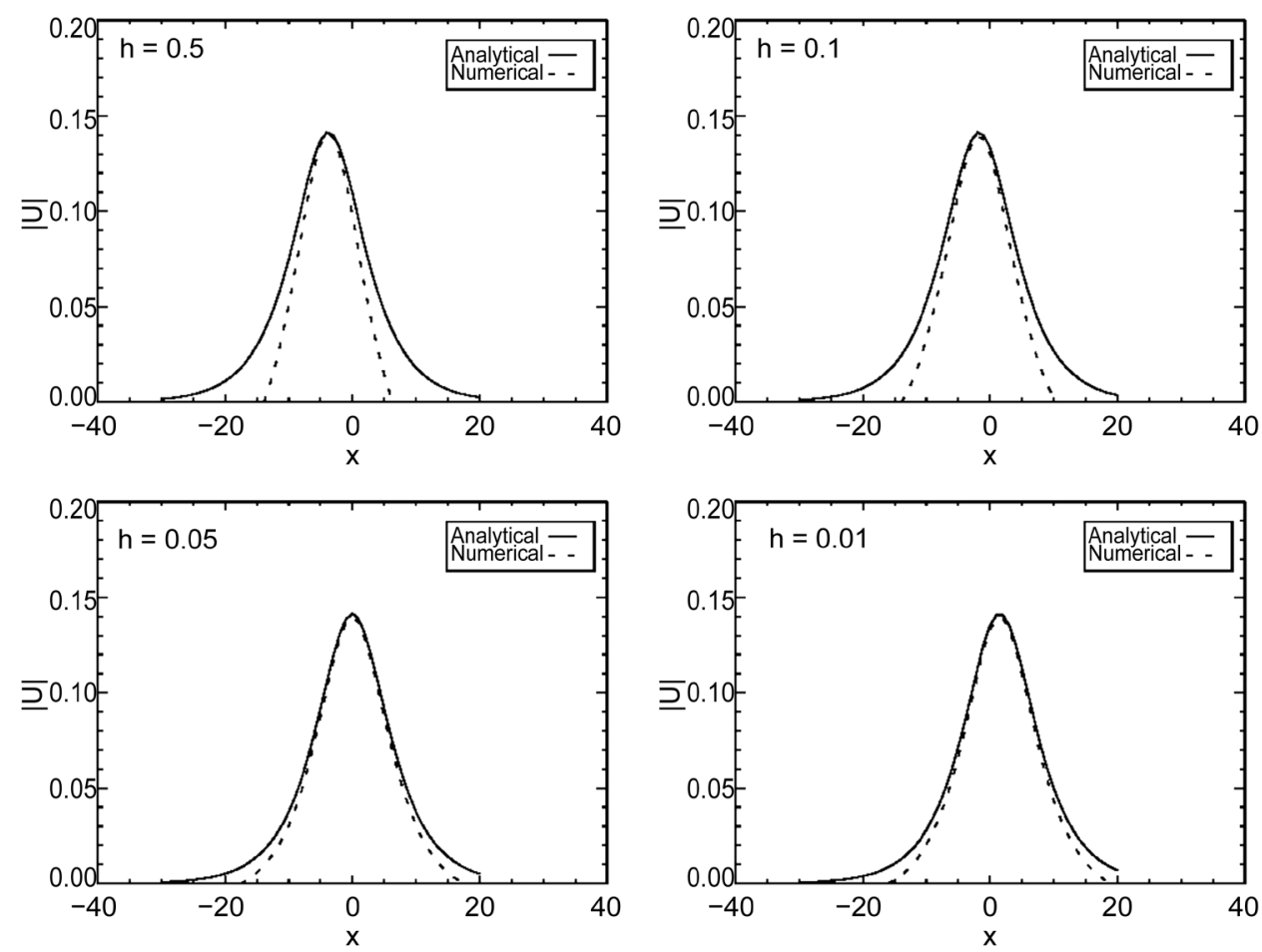

Figure 1. Comparison between the analytical solution (solid lines) and the numerical solution (dashed lines) of the 1D CNLS equation at different grid sizes at time-step size $\Delta t=0.05$ and time $t=21$. 
As shown in Figure 1, the numerical solution is continuous in the spatial domain, furthermore this solution is smoothly converging towards the analytical solution as the grid size deceases. It is also noted in Figure 1 that, at $h=0.5,0.1$ and 0.05 the numerical solution is converging more faster at small $|x|$; in the middle of the domain, than at large $|x|$; near the wall, meanwhile at $h=0.01$ this solution becomes relatively close to the physical one for all values of $|x|$.

In Figure 2, as it is clear the numerical solution is also continuous in the space domain, moreover this solution is gradually converging towards to the analytical solution as the time-step size decreases. It is also seen in Figure 2, at $\Delta t=0.25,0.125$ and 0.05 , the numerical solution is converging more slower at small $|x|$ than at large $|x|$, while at $\Delta t=0.01$ the numerical and the physical solution retains relatively similar.

As we mentioned before, the CNLS is time-dependent equation, because of this dependence, illustrating the efficiency of the FPSA at advanced time has to be undertaken. For this purpose, we re-conducted the comparison between the analytical and the numerical solution at different advanced times, the optimum grid and time-step size previously obtained in Figure 1 and Figure 2 are regarded in this comparison, and the result is plotted in Figure 3.

It is clear in Figure 3 that, the numerical solution is stable, and the most important, this solution perfectly matches the analytical one even at such a very long time.
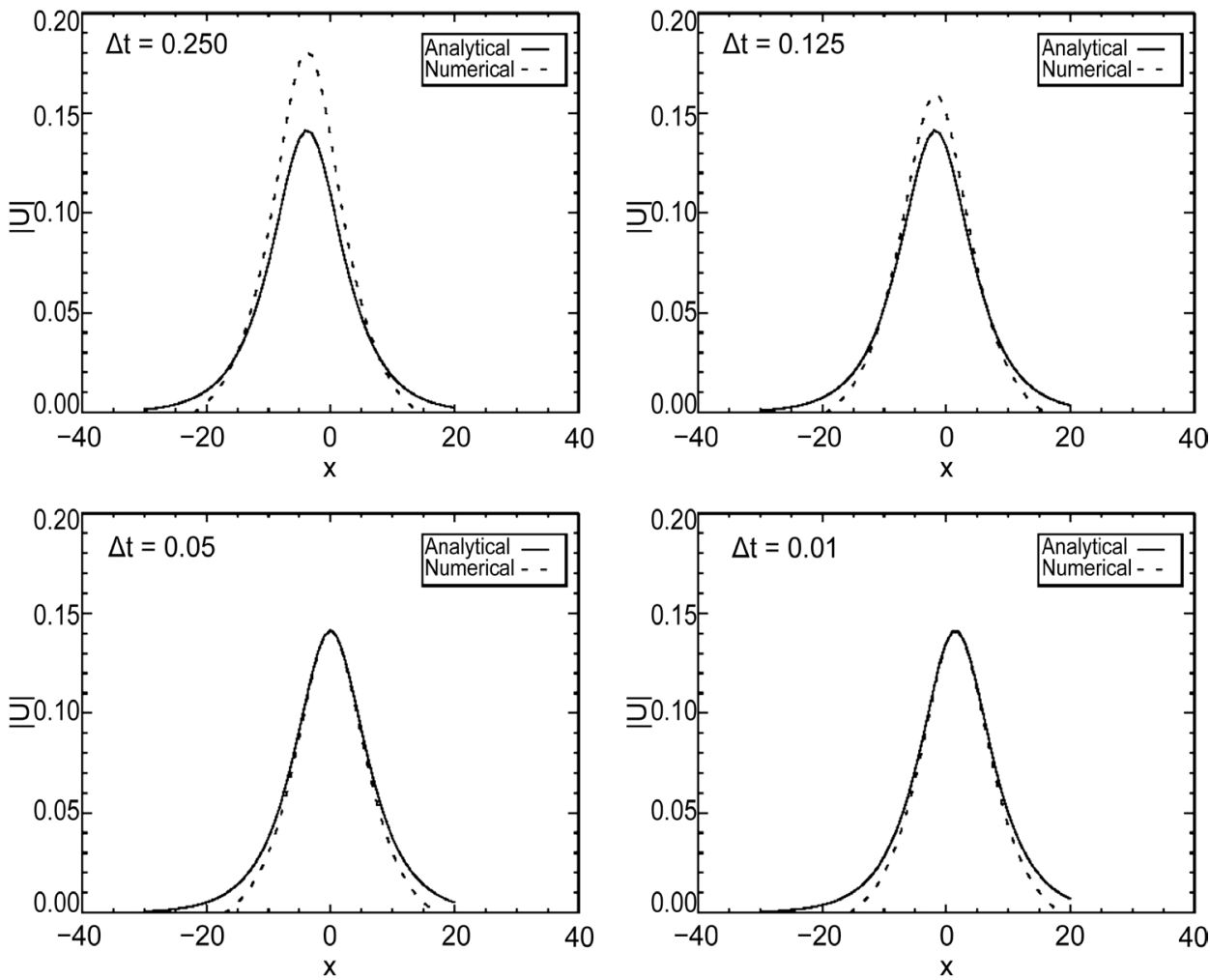

Figure 2. Comparison between the analytical solution (solid lines) and the numerical solution (dashed lines) of the 1D CNLS equation at different time-step sizes at grid size $h=0.01$ and time $t=17$. 

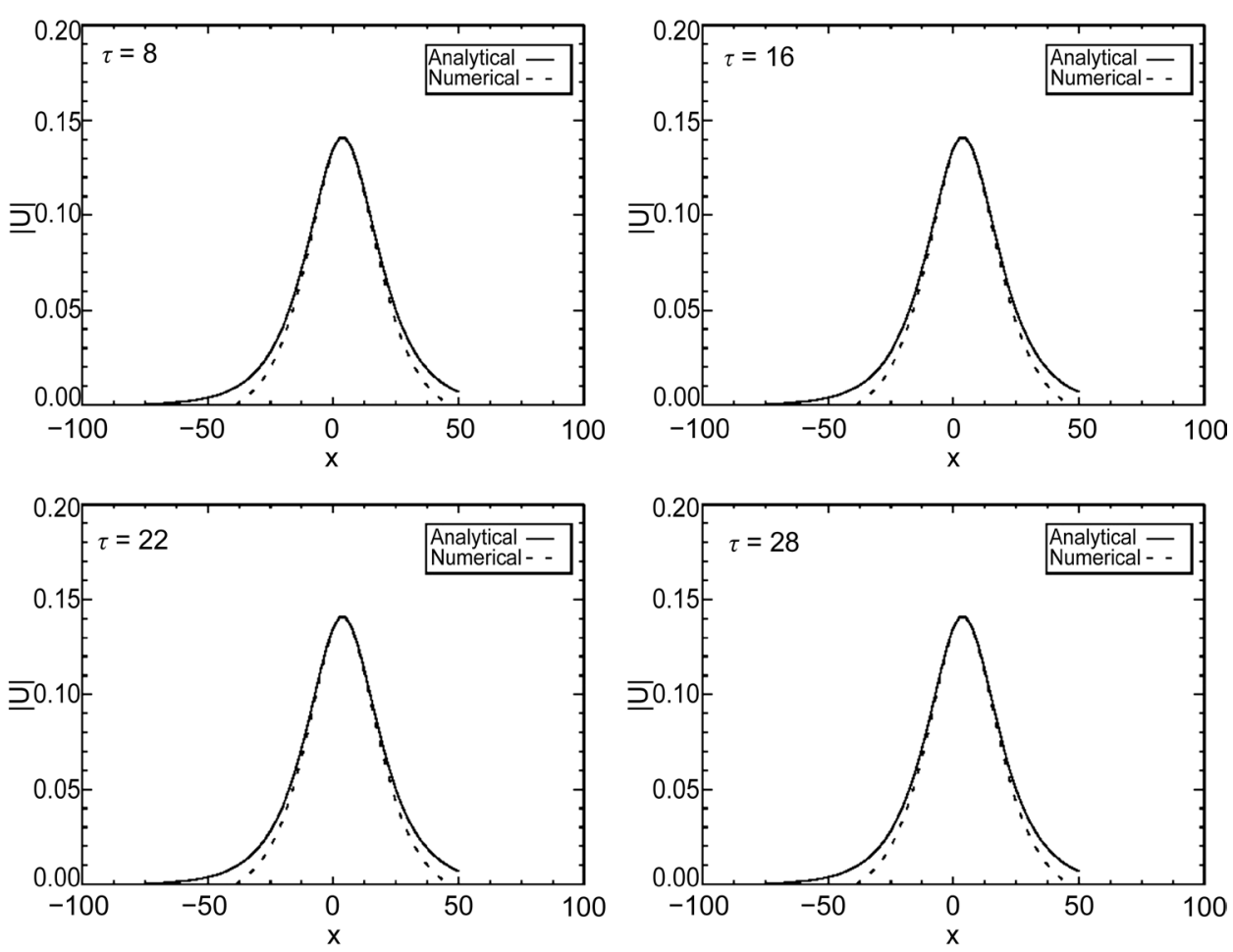

Figure 3. Comparison between the analytical solution (solid lines) and the numerical solution (dashed lines) of the 1D CNLS equation at different times at grid size $h=0.01$ and time-step size $\Delta t=0.01$.

\subsection{Determining the Accuracy}

The equation listed below is the Nonlinear Shrödinger Equation (NLSE) [20].

$$
i \frac{\partial \psi}{\partial t}=-\frac{1}{2}\left(\psi_{x x}+\psi_{y y}\right)+V(x, y) \psi+|\psi|^{2} \psi .
$$

The NLSE is a Gross-Pitaevskii equation that solves the Bose-Einstein condensate at a very low temperature. As noted above, the equation is $2 \mathrm{D}$ time-dependent, where $\psi$ is the wave function and $V(x, y)=\left(1-\sin ^{2} x \sin ^{2} y\right)$ is the trapping potential.

To conduct the present test, we applied the FPSA to numerically solve the NLSE in the spatial domain $[0,2 \pi] \times[0,2 \pi]$, where the following initial condition is applied:

$$
\psi_{0}(x, y)=\sin x \sin y \text {. }
$$

To determine the accuracy, we evaluated the absolute error $\left|\psi_{\text {exac }}(x, y, t)-\psi_{\text {num }}(x, y, t)\right|$ between the following exact solution:

$$
\psi_{\text {exac }}(x, y, t)=\sin x \sin y \exp (-2 i t),
$$

and the numerical solution $\psi_{\text {num }}(x, y, t)$ for $h=2 \pi / 128$ and $\Delta t=0.01$. The evaluated errors are listed in Table 1 at different computational times, and in order to perform a realistic benchmark comparison, we columned in the same table absolute errors of the Split-Step Finite Difference (SSFD) and the Split-Step Fourier Spectral (SSFS) methods that are previously applied to solve the 2D NLSE at the same grid size, time-step size, and computational times. 
Table 1. The absolute error of the SSFD, SSFS, and FPSA for 2D NLSE at $h=2 \pi / 128$ and $\Delta t=$ 0.01 .

\begin{tabular}{cccc}
\hline$t$ & SSFD & SSFS & FPSA \\
\hline 4 & $4.057 \mathrm{E}-4$ & $3.244 \mathrm{E}-12$ & $3.167 \mathrm{E}-12$ \\
8 & $8.115 \mathrm{E}-4$ & $1.250 \mathrm{E}-11$ & $2.821 \mathrm{E}-12$ \\
12 & $1.217 \mathrm{E}-3$ & $2.792 \mathrm{E}-11$ & $2.246 \mathrm{E}-12$ \\
16 & $1.623 \mathrm{E}-3$ & $4.951 \mathrm{E}-11$ & $1.853 \mathrm{E}-12$ \\
20 & $2.029 \mathrm{E}-3$ & $7.728 \mathrm{E}-11$ & $1.569 \mathrm{E}-12$ \\
24 & $2.434 \mathrm{E}-3$ & $1.113 \mathrm{E}-10$ & $8.334 \mathrm{E}-11$ \\
28 & $2.840 \mathrm{E}-3$ & $1.520 \mathrm{E}-10$ & $5.821 \mathrm{E}-11$ \\
32 & $3.246 \mathrm{E}-3$ & $2.008 \mathrm{E}-10$ & $3.774 \mathrm{E}-11$ \\
\hline
\end{tabular}

It is noted in Table 1 that: first, both of the FPSA and SSFS resolve such high accurate solution over the SSFD method; second, the FPSA retains a slightly higher accuracy than the SSFS as the computational time is advancing. The superiority of the FPSA and SSFS over the SSFD is a common feature for the most spectral methods, likely, it is now confirmed for the FPSA, for the second note, although the FPSA is slightly more accurate than the SSFS, in truth, the obtained accuracy degree is high enough to demonstrate a sufficiently accurate solution for the 2D NLSE and to preserve the physical behavior of this equation at a long evolution time.

\section{Numerical Experiments}

After we illustrated the efficiency and determined the accuracy of the FPSA in the previous section, herein we examine its performance. To examine this performance, we carry out a number of numerical experiments [23] to study selected phenomena in laser interactions in plasma, these phenomena are the self-focusing, the multiple filamenttation, and the periodic self-focusing and defocussing of a femtosecond laser beam in air plasma.

In these experiments, we apply the FPSA to numerically solve the envelope-equation in the transverse plane ( $x-y$ plane) for an incident linearly polarized laser beam with an initial Gaussian profile $a=a_{0} \mathrm{e}^{-\left(x^{2}+y^{2}\right) / r_{o}^{2}}$; where $a_{0}$ is the initial complex amplitude and $r_{0}=1 \mu \mathrm{m}$ is the spot size. Also in these experiments, to consider the QSA, the speed of light frame is used through the following transformation:

$$
(x, y, z, t) \rightarrow(x, y, \eta, \tau) ;
$$

where $\eta=z-k_{0} c / \omega_{p e} t, k_{0}$ is the wave number of the applied beam, $c$ is the speed of light, and $\omega_{p e}$ is plasma frequency, and to work under the paraxial approximation, $\partial / \partial \eta=0$ is assumed. Moreover, in our experiments the time $\tau$ is normalized by $\omega_{p e}^{2} t / \omega_{0}$, the complex amplitude $a$ is normalized by $m c^{2} / e$; where $m$ and $e$ are the electron mass and charge, receptively, the co-ordinates $x$ and $y$ are normalized by $c / \omega_{p e}$, the electron density $n$ is normalized by the unperturbed density $n_{0}$, and the 
power $P$ is normalized by the critical power for self-focusing $P_{c r}$.

\subsection{The Self-Focusing}

The self-focusing or channeling is a basic nonlinear phenomenon in laser interactions in plasmas [24], two different self-focusing processes due to two separate effects are demonstrated in this interaction, namely the ponderomotive self-focusing (PSF) and relativistic self-focusing (RSF). The PSF is a contribution to an induced ponderomotive force, while RSF emerges in the presence of an applied super-intense Gaussian laser beam, in the present experiment we study PSF to examine the FPSA performance.

To study the PSF, we presented in Figure 4 the absolute-amplitude $|a|$ of a laser beam at different simulation times, the initial complex amplitude of this beam is given in this figure. Note that, the given amplitude is selected to be sufficient enough to demonstrate the PSF and below the relativistic limit to avoid the RSF. As shown in Figure 4, at $\tau=60$ a peak beam intensity with a relatively small spot-size that is highly localized around its initial centroid is clearly observed. We point out that, the observed structure is the self-focusing in the formation stage, and in order to preciously study the PSF, we have to follow the evolution of this structure at different times. At $\tau=75$, the self-focusing structure is significantly enhanced, as displayed, its intensity is more focused and its spot-size is more narrowed. The determined enhancement in both of the intensity and spot-size of the self-focusing structure is known as the self-focusing developing, in reality, the self-focusing developing is an effective stage in the PSF process, since during this stage the focused-intensity is being much intensified and the spot-size is getting much more narrowed as seen at $\tau=85$. Next at $\tau=95$, neither increase in the focused-intensity nor decrease in the spot size is observed in the structure, according to this observation we can say that the self-focusing developing is halted, but since this static behavior of the invariant focused-intensity and the constant spot-size is extended further as seen at $\tau=120$, one can conclude that the self-focusing is stabilized. The stabilized self-focusing stage is crucially necessary in the diagnostics and wide range of laser plasmas applications, however, this stage can be remained longer or vanished shortly depending on the balance of the PSF with the plasma ionization.

\subsection{The Multiple Filamentation}

In laser interactions in plasmas, when the power of a focused beam exceeds the critical value for beam collapse $\left(P_{c}\right)$ in the presence of a spatial-temporal perturbation on this beam profile, the focused beam is suddenly collapsed and turned into narrow patterns of small filaments. This process is called the Multiple Filamentation (MF). Understanding the MF phenomena is essential in the supercontium radiation production and lighting control, from that point on, the MF is an extremely interested phenomenon to be studied.

To study the MF phenomena, we presented in Figure 5 the transverse filamentation dynamics of the absolute amplitude $|a|$ of a laser beam at initial complex amplitude given in this figure. We have to keep in mind that, the initial amplitude given in this 

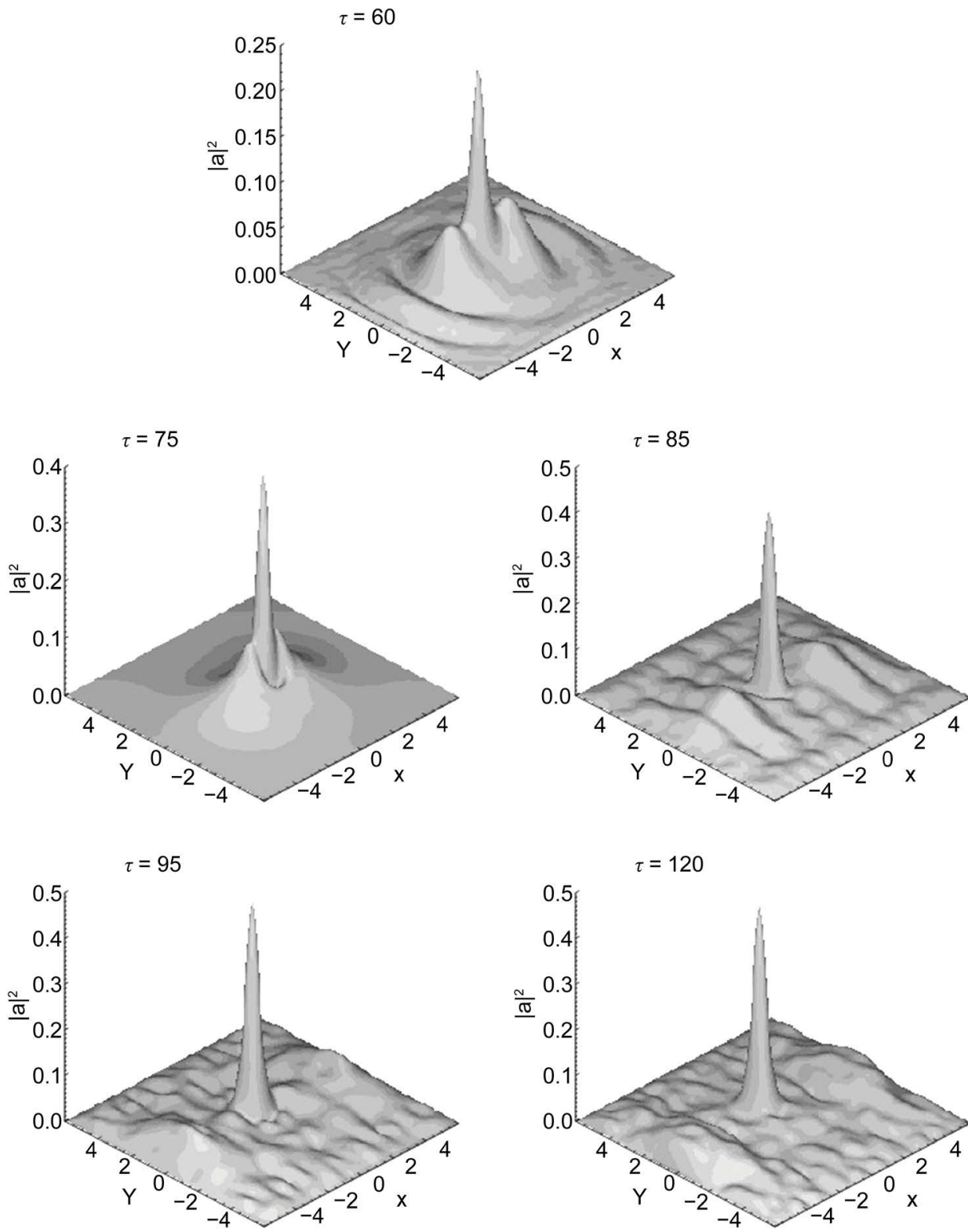

Figure 4. The absolute amplitude $|a|$ of a laser beam at initial complex amplitude $a_{0}=0.15$ at different simulation times.

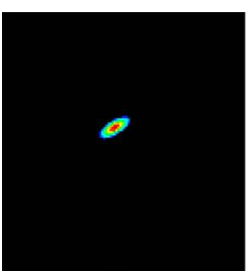

(a)

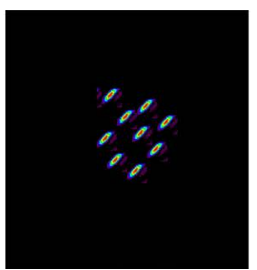

(b)

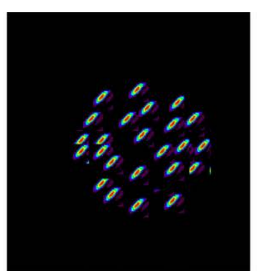

(c)

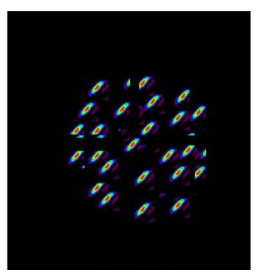

(d)

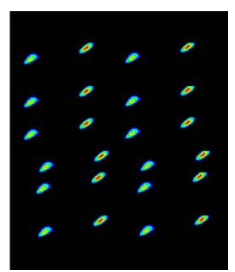

(e)

Figure 5. The transverse filamentation dynamics of the absolute amplitude $|a|$ of a laser beam at initial complex amplitude $a_{0}=0.4$ at different simulation times. $\tau=50$ in (a), $\tau=55$ in (b), $\tau=65$ in (c), $\tau=90$ in (d), and $\tau=120$ in (e). 
figure is selected to be high enough not only to demonstrate the self-focusing, but to reach a focused power above the critical power for collapse. As shown in Figure 5, at $\tau=$ 50 , a highly localized self-focused spot is seen, shortly at $\tau=55$, this spot is collapsed into narrow filaments. The collapse is demonstrated due to the reason explained before, even though, this collapse is carrying on as long as each of these filaments consumes a power $P>P_{\mathcal{o}}$ thus at $\tau=65$, the narrow filaments are more collapsed and its number is more increased. Later at $\tau=90$, the filaments are stagnate, as no more collapse is observed beside the filaments number remains approximately constant. Owing to the fact that, these stagnated filaments are a subject to various dynamics; depending on the distortion on the initial beam profile and the period between the beam shots, in examples including the mutual attraction where the separation-distance among the filaments deceases until the filaments are fused, or the mutual repulsion where this separationdistance increases and consequently the filaments are more widely spread. In our result, it is clear that the mutual repulsion is the most dominated dynamics, as seen at $\tau=120$ the narrow filaments run away from one another and spatially spread. Although the mutual repulsion would reduce the effectiveness of generation of strong filaments, this dynamics gives a better understanding for the physics of the soliton vortices and spiraling.

\subsection{The Periodic Self-Focusing and Defocussing}

The filamentation of a femtosecond (fs) laser in air plasma [7] is the most modern and rapidly developing research topic in laser interactions in plasma. In this topic, the formation of a fs filament that stably propagates over a long propagation-distance is compulsory for particular applications [25], such as Light Detection and Ranging (LiDAR), remote sensing of atmospheric pollution, pulse compression, electric charge triggering and guiding, and remote Terahertz pulse generation. Among various phenomena associated with the filamenation process, the periodic self-focusing and defocussing is the principle phenomena that exposes the filament formation, and combined with other dynamics at specific input beam parameters, this phenomena can stably control the properties of the formed filament over a lengthen distance. In this experiment, we apply the FPSA to simulate the periodic self-focusing and defocussing of a fs laser filamenttaion in air plasma.

To simulate the periodic self-focusing and defocussing, we applied a 1D FPSA to solve the envelope-equation. This is simply because this phenomena is a $1 \mathrm{D}$ dynamics which demonstrates along the direction of propagation $\left(z^{-}\right)$only. In addition, in this simulation we considered the fs parameters [26], particularly the pulse duration $f=$ $10^{-15}$, the wavelength $\lambda=800 \mathrm{~nm}$, the spot size $r_{0}=17 \mathrm{~mm}$, and the critical power $P_{c r}=$ 3.32 GW, the simulation results are presented in Figure 6 and Figure 7.

Figure 6 shows the filamentaion of a fs laser beam in air plasma at initial input power $P_{0}=200 P_{c r}$ as seen in this figure, a periodically unbalanced and instable fs filament is clearly formed, as noted the defocsuing period of this filament is longer than the self-focusing term, and at an advanced propagation-distance the formed filament is 


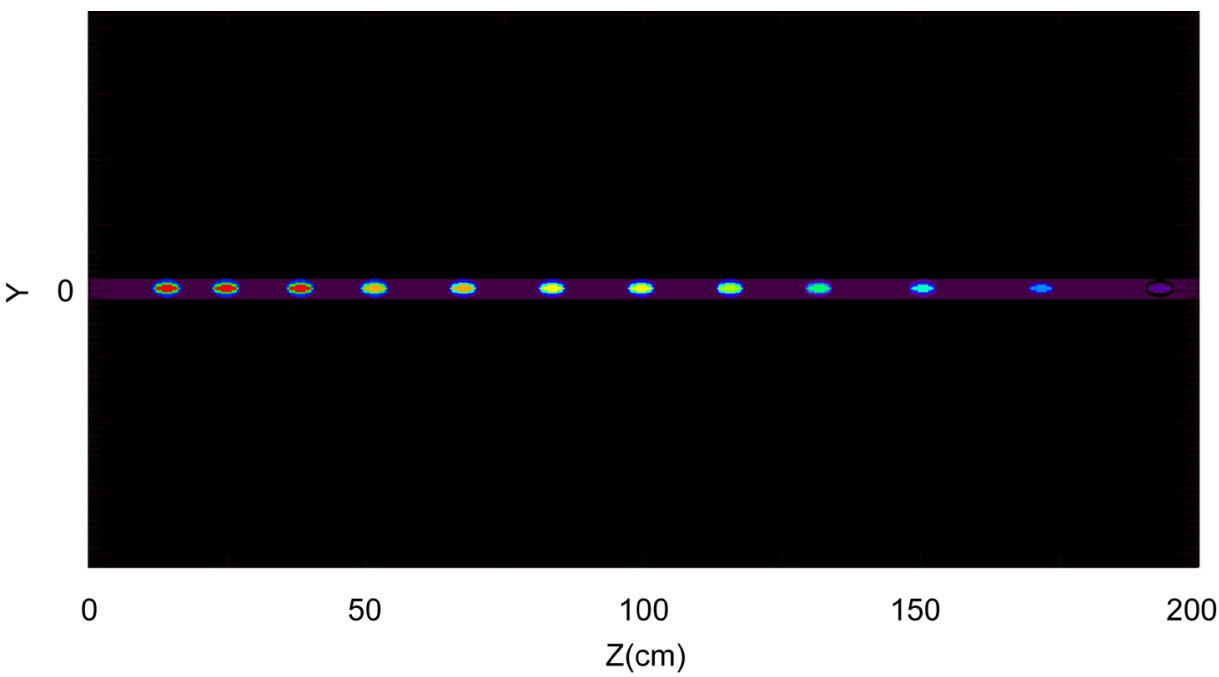

Figure 6. The filamentation of a fs laser in air plasma at initial input power $P_{0}=200 P_{c r}$.

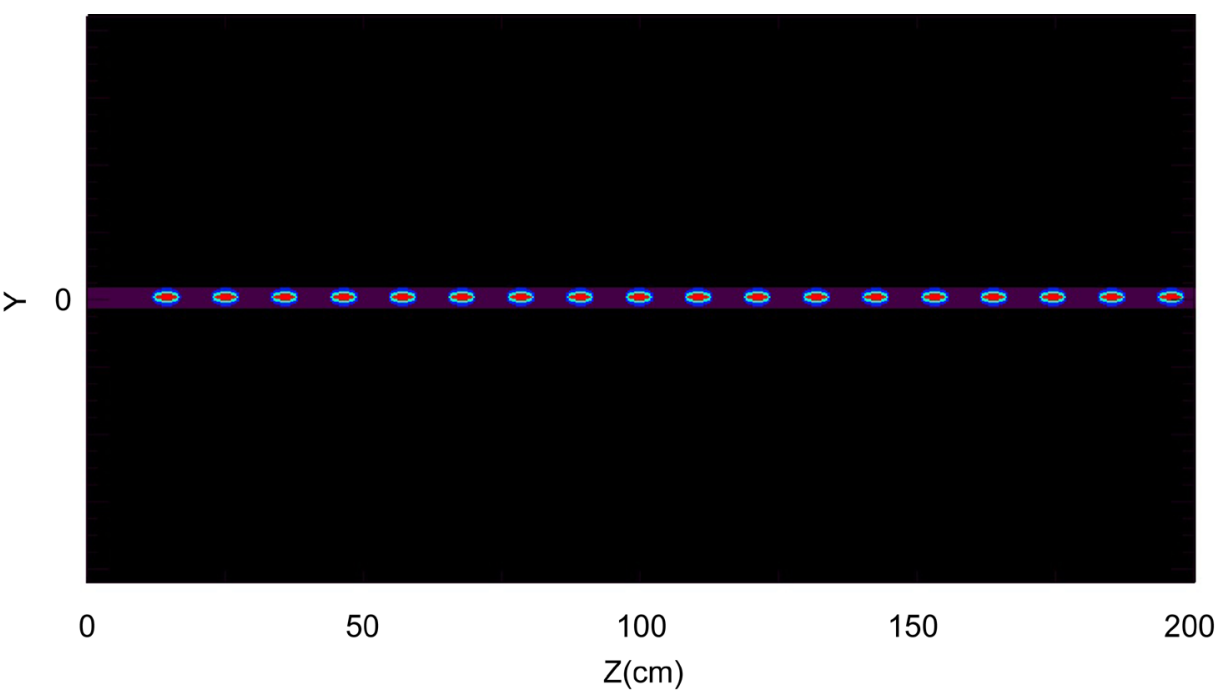

Figure 7. The filamentation of a fs laser in air plasma at initial at input power $P_{0}=500 P_{c r}$.

gradually decayed and finally disappeared. The balance between the self-focusing and defocsuing period is subject to the balance between the filament intensity-converging rate during the self-focusing period and the intensity-diverging rate in the defocussing one, as long as these two rates are comparable, a periodically balanced self-focusing and defocussing periods can be achieved, otherwise, unsatisfactory results as seen in Figure 6 are obtained. Since the two rates are input power dependence, we repeated the previous simulation at another input power, and the result is shown in Figure 7.

Figure 7 shows the filamentaion of fs laser in air plasma at input power $P_{0}=500 P_{c r}$, as shown in this figure, a fs filament with periodically balanced self-focused and defocsued periods that stably propagates over a long propagation-distance is clearly formed. In the fs filamentation in air plasma, the stable propagation-distance is changeable from application to another; depending on the input parameters, in our experiment a stable 
propagation-distance of few meters is satisfactory.

\section{Conclusions}

We successfully applied a Fourier pseudospectral algorithm to solve a 2D nonlinear paraxial envelope-equation of laser interactions in plasmas. In this algorithm, we used the second order Strang time-splitting method to split the envelope-equation into three equations; two ordinary differential equations where the source term is included and one 2D partial differential equation (PDE) where the spatial operators only are existing. To obtain the solution, we spatially discreted the field quantity and its spatial derivatives in term of the Fourier polynomials, and then we integrated the resultant equations sequentially using the discrete time integration.

We illustrated the efficiency and determined the accuracy of the proposed algorithm in two numerical tests. In the first test, the algorithm was shown to be valid for solving the 1D Coupled Nonlinear Schrödinger Equation (CNLS) and sufficiently efficient to present stable and accurate results over a sufficiently long computational time. In the second test, the evaluated absolute error confirmed that the algorithm provides sufficiently accurate solution for the 2D Nonlinear Schrödinger Equation (2D NLSE) and compares more accurately with other available schemes.

We examined the performance of the algorithm in a series of numerical experiments. In these experiments, the algorithm showed considerable potential for studying fundamental and advanced phenomena in laser interactions in plasmas. At low input laser intensity, the algorithm efficiently depicts the ponderomotive self-focusing formation and smoothly follows the self-focusing developing and stabilization. Furthermore, at a focused power over the critical power for self-focusing, the algorithm clearly images the sudden self-focused beam collapse and the multiple filaments formation. Moreover, by tunning the input power of an input femtosecond beam, the FPSA successfully simulates the formation of a fs filament that stably propagates over a long propagationdistance in air plasma.

\section{References}

[1] Hafizi, B., Sprangle, P. and Ting, A. (1995) Envelope Description of Electron Beams in Plasmabased Accelerators. AIP Conference Proceedings, 335, 480-500. https://doi.org/10.1063/1.48288

[2] Litvak, A.G. (1970) Finite-Amplitude Wave Beams in Magnetoactive Plasma. Soviet Physics-JETP, 30, 344.

[3] Pajouh, H.H., Abbasi, H. and Shukla, P.K. (2004) Nonlinear Interaction of a Gaussian Intense Laser Beam with Plasma: Relativistic Modulational Instability. Physics of Plasmas, 11, 5697-5703. https://doi.org/10.1063/1.1810159

[4] McKinstrie, C.J. and Bingham, R. (1989) The Modulational Instability of Coupled Waves. Physics of Fluids B, 1, 230-237. https://doi.org/10.1063/1.859095

[5] Esarey, E., Sprangle, P., Krall, J. and Ting, A. (1996) Overview of Plasma-Based Accelerator Concepts. IEEE Transactions on Plasma Science, 24, 252-288.

https://doi.org/10.1109/27.509991 
[6] Ganeev, R.A., Suzuki, M., Baba, M. and Kuroda, H. (2007) High-Order Harmonic Generation from Laser Plasma Produced by Pulses of Different Duration. Physical Review A, 76, Article ID: 023805. https://doi.org/10.1103/PhysRevA.76.023805

[7] Couairon, A. and Mysyrowicz, A. (2007) Femtosecond Filamentation in Transparent Media. Physics Report, 441, 47-189. https://doi.org/10.1016/j.physrep.2006.12.005

[8] Namiki, T. (1999) A New FDTD Algorithm Based on Alternating-Direction Implicit Method. IEEE Transactions on Microwave Theory and Techniques, 47, 2003-2007. https://doi.org/10.1109/22.795075

[9] Mahdy, A.I. (2008) Interactions of Two Co-Propagating Laser Beams in Underdense Plasmas Using a Generalized Peaceman-Rachford ADI Form. Computer Physics Communications, 178, 249-271. https://doi.org/10.1016/j.cpc.2007.09.002

[10] Elkin, N., Napartovich, A., Sukharev, A., Troschieva, V. and Vysotsky, D. (2000) Direct Numerical Simulation of Radiation Propagation in a Multicore Fiber. Optics Communications, 177, 207-217. https://doi.org/10.1016/S0030-4018(00)00556-3

[11] Huang, C., et al. (2006) Quickpic: A Highly Efficient Particle-in-Cell Code for Modeling Wakefield Acceleration in Plasmas, Journal of Computational Physics, 217, 658-679. https://doi.org/10.1016/j.jcp.2006.01.039

[12] Hur, M.S. and Suk, H. (2007) New Envelope-Kinetic Scheme for the Simulation of Raman Backward Laser Amplification. Computer Physics Communications, 177, 68-69. https://doi.org/10.1016/j.cpc.2007.02.086

[13] Malkin, V.M., Shvets, G. and Fisch, N.J. (2000) Ultra-Powerful Compact Amplifiers for Short Laser Pulses. Physics of Plasmas, 7, 2232-2240. https://doi.org/10.1063/1.874051

[14] Boyd, J. (2001) Chebyshev and Fourier Spectral Methods. 2nd Edition, Dover, New York.

[15] Chen, Y., Song, S. and Zhu, H. (2011) The Multi-Symplectic Fourier Pseudospectral Method for Solving Two-Dimensional Hamiltonian PDEs. Journal of Computational and Applied Mathematics, 236, 1354-1369. https://doi.org/10.1016/j.cam.2011.08.023

[16] Muslu, G. and Erbay, H. (2005) Higher-Order Split-Step Fourier Schemes for the Generalized Nonlinear Schrödinger Equation. Mathematics and Computers in Simulation, 67, 581595. https://doi.org/10.1016/j.matcom.2004.08.002

[17] Bao, W. and Shen, J. (2008) A Generalized-Laguerre-Hermite Pseudospectral Method for Computing Symmetric and Central Vortex States in Bose-Einstein Condensates. Journal of Computational Physics, 227, 9778-9793. https://doi.org/10.1016/j.jcp.2008.07.017

[18] Bao, W., Sun, F. and Wei, G. (2003) Numerical Methods for the Generalized Zakharov System. Journal of Computational Physics, 190, 201-228. https://doi.org/10.1016/S0021-9991(03)00271-7

[19] Sprangle, P., Esarey, E. and Ting A. (1990) Nonlinear Theory of Intense Laser-Plasma Interactions. Physical Review Letters, 64, 2011-2014.

https://doi.org/10.1103/PhysRevLett.64.2011

[20] Wang, H. (2005) Numerical Studies on the Split-Step Finite Difference Method for Nonlinear Schrödinger Equations. Applied Mathematics and Computation, 170, 17-35. https://doi.org/10.1016/j.amc.2004.10.066

[21] Weizhu, B., Shi, J. and Peter, A. (2002) On Time-Splitting Spectral Approximations for the Schrödinger Equation in the Semiclassical Regime. Journal of Computational Physics, 175, 487-524. https://doi.org/10.1006/jcph.2001.6956

[22] Sanz-Serna, J.M. and Verwer, J.G. (1986) Conerservative and Nonconservative Schemes for the Solution of the Nonlinear Schrödinger Equation. IMA Journal of Numerical Analysis, 6, 
25-42. https://doi.org/10.1093/imanum/6.1.25

[23] Press, W.H., Teukolsky, S.A., Vetterling, W.T. and Flannery, B.P. (1996) Numerical Recipes in Fortran 90: The Art of Parallel Scientific Computing. 2nd Edition, Cambridge University Press, Cambridge.

[24] Brandi, H.S., Manus, C., Mainfray, G., Lehner, T. and Bonnaud, G. (1993) Relativistic and Ponderomotive Selffocusing of a Laser Beam in a Radially Inhomogeneous Plasma. i. Paraxial Approximation. Physics of Fluids B: Plasma Physics, 5, 3539-3550. https://doi.org/10.1063/1.860828

[25] Chin, S.L., et al. (2012) Advances in Intense Femtosecond Laser Filamentation in Air. Laser Physics, 22, 1-53. https://doi.org/10.1134/S1054660X11190054

[26] Deng, Y., Jin, T., Zhao, X., Gao, Z. and Chi, J. (2013) Simulation of Femtosecond Laser Pulse Propagation in Air. Optics \& Laser Technology, 45, 379-388.

http://www.sciencedirect.com/science/article/pii/S0030399212002885

https://doi.org/10.1016/j.optlastec.2012.06.020

Submit or recommend next manuscript to SCIRP and we will provide best service for you:

Accepting pre-submission inquiries through Email, Facebook, LinkedIn, Twitter, etc.

A wide selection of journals (inclusive of 9 subjects, more than 200 journals)

Providing 24-hour high-quality service

User-friendly online submission system

Fair and swift peer-review system

Efficient typesetting and proofreading procedure

Display of the result of downloads and visits, as well as the number of cited articles

Maximum dissemination of your research work

Submit your manuscript at: http://papersubmission.scirp.org/

Or contact jamp@scirp.org 